\title{
A rare cause of neurological deterioration to complete paraplegia after surgery for thoracic myelopathy: a case report
}

\author{
Kalyan Kumar Varma Kalidindi ${ }^{1}$ Mayank Gupta ${ }^{1} \cdot$ Harvinder Singh Chhabra ${ }^{1}$
}

Received: 4 March 2019 / Accepted: 16 May 2019

(c) International Spinal Cord Society 2019

\begin{abstract}
Introduction Progressive deterioration of neurological status post-thoracic myelopathy surgery after a clinically stable period is rare and can pose a diagnostic dilemma. We present our experience with such a case where all known etiologies were ruled out and the cause of deterioration could not be conclusively identified. The course was found to be similar to subacute post-traumatic ascending myelopathy (SPAM). However, the condition has only been described for traumatic injuries so far.

Case presentation Our patient presented a history of back pain and associated gait instability for one and a half months. There was no history of trauma. Investigations suggested an Anderson-like lesion at T11-T12 with cord edema at the same level suggestive of instability. She underwent posterior stabilization T9 to L2 and laminectomy of T11 as well as T12 under neuromonitoring. The postoperative sequence of events included an episode of pyrexia on the fifth day of surgery, neurological deterioration from the seventh day of surgery proceeding to complete paraplegia by the fourteenth day, no response to steroid treatment and no signs of recovery till two years post surgery. MRI findings were suggestive of SPAM, and there was no evidence of infection.

Discussion Ascending myelopathy is a potential but rare cause of delayed deterioration in neurological status after surgical intervention. MRI findings of cord edema extending more than four levels above the involved segments is a characteristic finding of the condition. Ascending myelopathy may lead to complete cord injury. The precise cause of the condition is unknown and prognosis remains poor.
\end{abstract}

\section{Introduction}

Outcomes of surgical management of thoracic myelopathy have not been very promising. One of the main contributing factors to the poor outcome is the vulnerable spinal cord at the site of the lesion due to relative avascularity and reduced available space [1]. The deterioration of neurological status is a known complication of surgical decompression of thoracic myelopathy detected, either intraoperatively through neuromonitoring or in the immediate postoperative period on assessment of neurological status [2].

Kalyan Kumar Varma Kalidindi

kalyanvarmambbs@gmail.com

kalyan_varma_mbbs@yahoo.com

1 Department of Spine Service, Indian Spinal Injuries Center, New Delhi, India
Progressive deterioration of neurological status postoperatively is rare and can pose a diagnostic dilemma. We present our experience with one such case where all known etiologies were ruled out and the cause of progressive postoperative neurological deficit could not be conclusively identified. On thorough search of the available literature, the clinical course was found to most closely resemble an entity known as SPAM, which has so far been reported to occur only after significant trauma. The rarity of occurrence and lack of any published article till date warrants this case to be reported in the literature.

\section{Case presentation}

A 43-year-old female was presented to our center with severe pain of 6 weeks' duration in the mid-back. The pain was insidious in onset, continuous, of progressively increasing intensity and with a postural variation. She was unable to lie supine due to severe intercostal pain and had to 

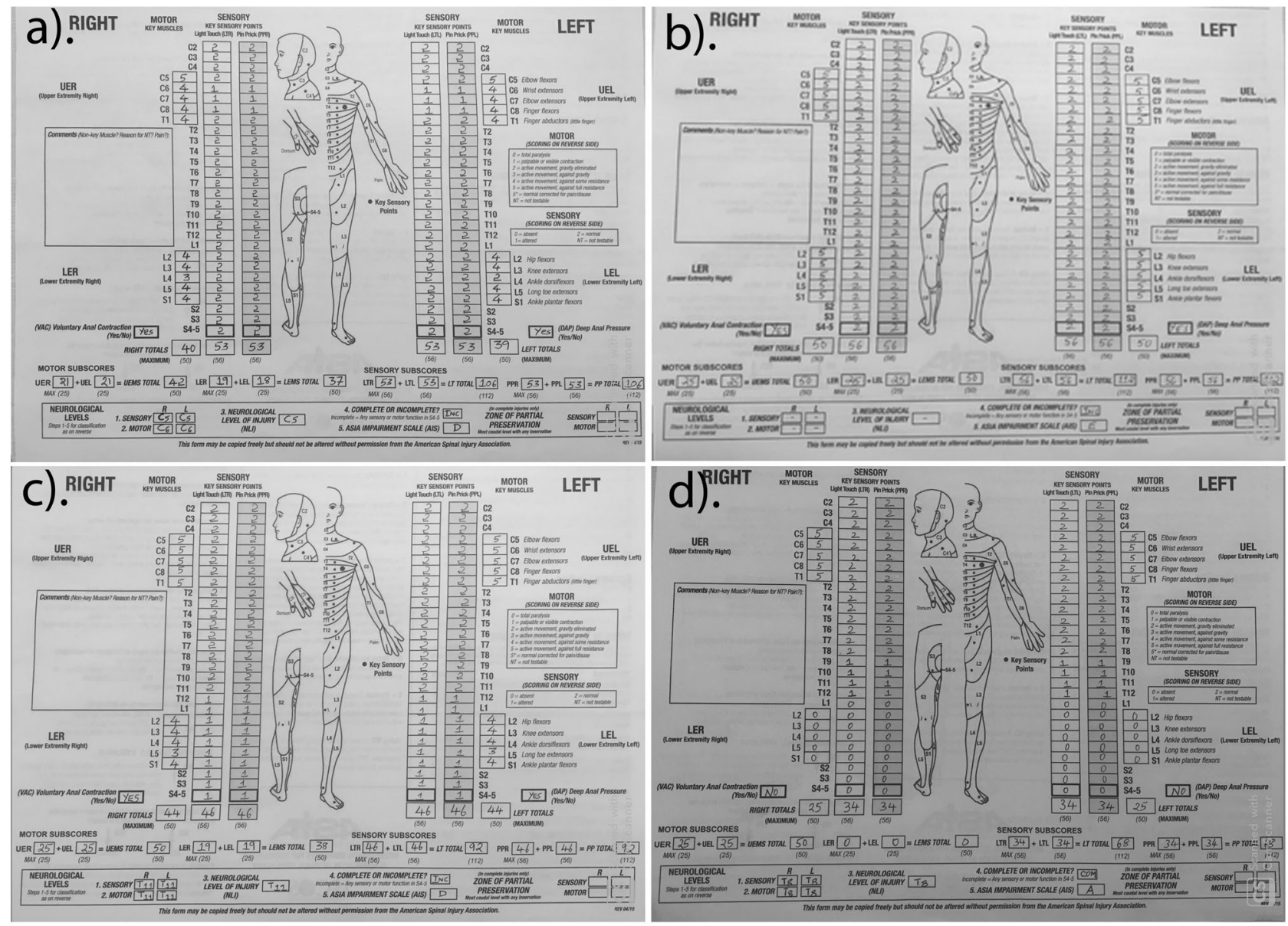

Fig. 1 The neurologic status as per ASIA/ISCoS-ISNCSCI (American Spinal Injury Association/International Spinal Cord SocietyInternational Standards for Neurological Classification of Spinal Cord Injury). a At the time of earlier presentation with symptoms of cervical myelopathy 2 years ago. b At 1 year follow up period after surgery for cervical myelopathy by posterior instrumentation $\mathrm{C} 1-\mathrm{T} 1$

sleep in a sitting posture causing severe disability. There were associated non-dermatomal paresthesias in both lower limbs. She had been bedridden for the past 4 weeks before the presentation due to severe gait instability, resulting in an inability to walk. There were no bowel or bladder symptoms. There was no history of associated fever, significant trauma, constitutional symptoms or history suggestive of an inflammatory etiology. She had a past history suggestive of compressive cervical myelopathy two years ago when she was presented to us with difficulty in walking and weakness in both upper limbs. Her neurologic status at that time of presentation was attached in Fig. 1a according to the ASIA/ ISCoS-ISNCSCI (American Spinal Injury Association/ International Spinal Cord Society-International Standards for Neurological Classification of Spinal Cord Injury). Posterior stabilization from $\mathrm{C} 1$ to $\mathrm{T} 1$ and decompression by cervical laminectomy $\mathrm{C} 1$ to $\mathrm{C} 7$ and foramen magnum decompression was done at our Center. She had a good and $\mathrm{C} 1$ to $\mathrm{C} 7$ decompression along with foramen magnum decompression. c At the time of presentation with symptoms of thoracic myelopathy. d At 2 weeks post surgery for thoracic myelopathy by posterior instrumented fusion from T9 to L2 vertebrae and decompression $\mathrm{T} 11-\mathrm{T} 12$

outcome with an improvement of symptoms and regained the ability to walk without support (Fig. 1b).

On examination, there was tenderness in the mid-back over T11-T12 region. Movements of the spine could not be tested due to severe pain. Higher mental functions, cranial nerve functions and neurological status in the upper limbs were within normal limits. In both lower limbs, there was increased tone and weakness. The neurologic status was as attached in Fig. 1c. Deep tendon reflexes in knee and ankle were exaggerated and plantar reflexes were extensor bilaterally.

On the basis of clinical findings, a provisional diagnosis of thoracic myelopathy was made. Radiological examination revealed multiple syndesmophytes with suspected instability between $\mathrm{T} 11$ and $\mathrm{T} 12$ vertebrae on standing anteroposterior and lateral radiographs with features suggestive of Diffuse Idiopathic Skeletal Hyperostosis (DISH). 
Fig. 2 Preoperative sagittal (a) and axial (b) T2 MRI images of the patient's thoracolumbar spine suggestive of a discovertebral lesion at T11-T12 with instability with cord edema at the level of the lesion. Preoperative T2 MRI screening of the whole spine (c) showing no lesion above and below the area of interest
Fig. 3 Postoperative sagittal (a) and axial (b) T2 MRI images of the thoracolumbar spine taken 2 days after surgery show no extension of edema. Artifacts were caused by the pedicle screw and rod instrumentation used for stabilization.

Postoperative X-rays (c) of the patient's thoracolumbar spine showing proper placement of screws and stabilization of the lesion. CT scan (d) of the thoracolumbar spine was obtained to exclude possible spinal canal compromise by the instrumentation
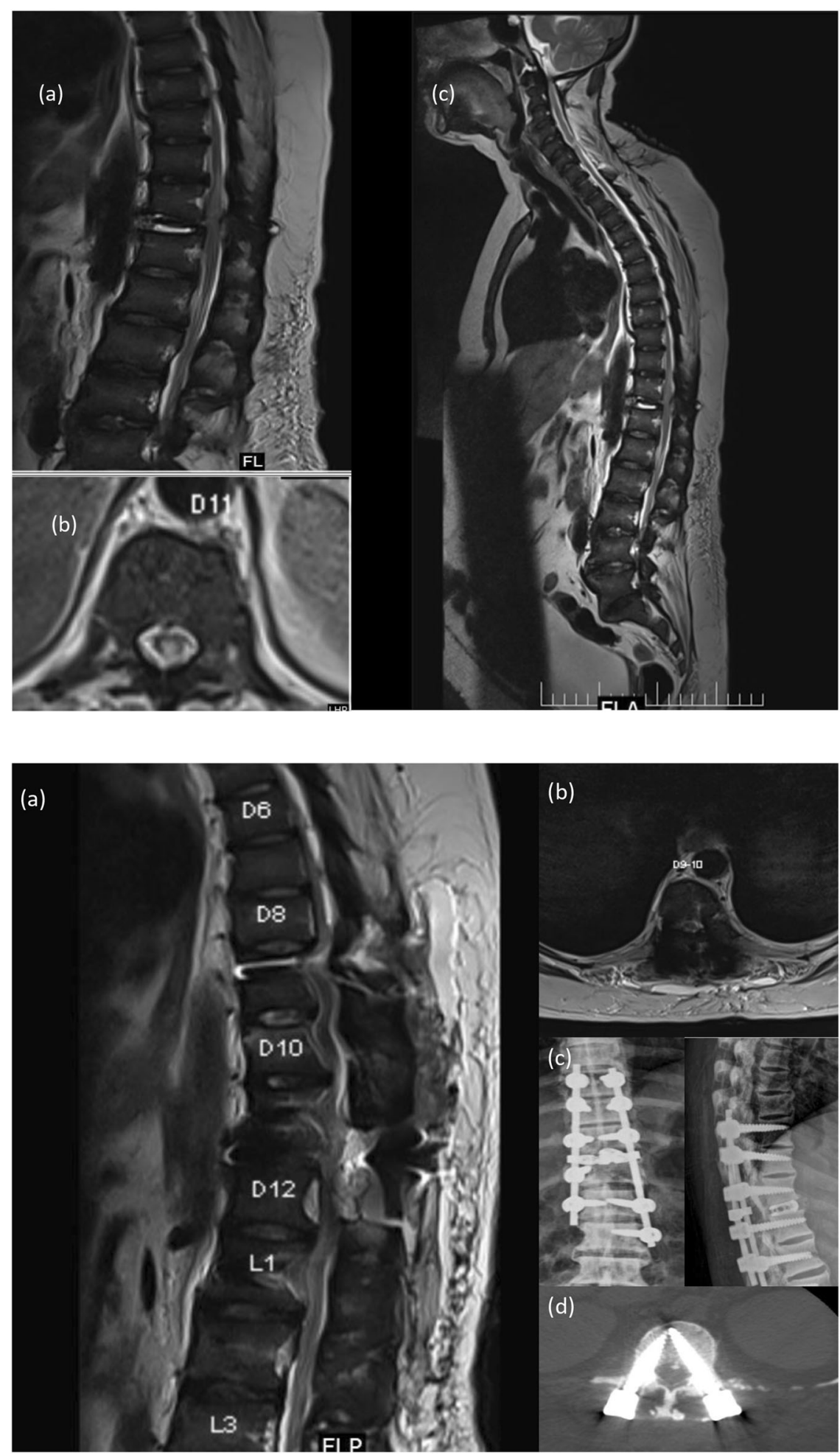

The patient could not lie supine in MRI due to severe back as well as girdle pain and hence, an MRI was done under sedation. It revealed a three-column Anderson-like lesion at T11-T12 level with cord edema behind T11 vertebra suggestive of dynamic cord compression (Fig. 2).
Based on these clinical and radiological findings, the patient was advised stabilization and decompression under general anesthesia to reduce pain and prevent further deterioration in neurological status. After a thorough preoperative workup, preanesthetic checkup and written 
Fig. 4 Sagittal (a) and axial $(\mathbf{b}, \mathbf{c})$ postoperative T2 MRI images of the patient's thoracolumbar spine taken 2 weeks after surgery showing the extension of edema (marked by blue arrow) up to $\mathrm{T} 7$ level. Artifacts are caused by the pedicle screw and rod instrumentation used for stabilization

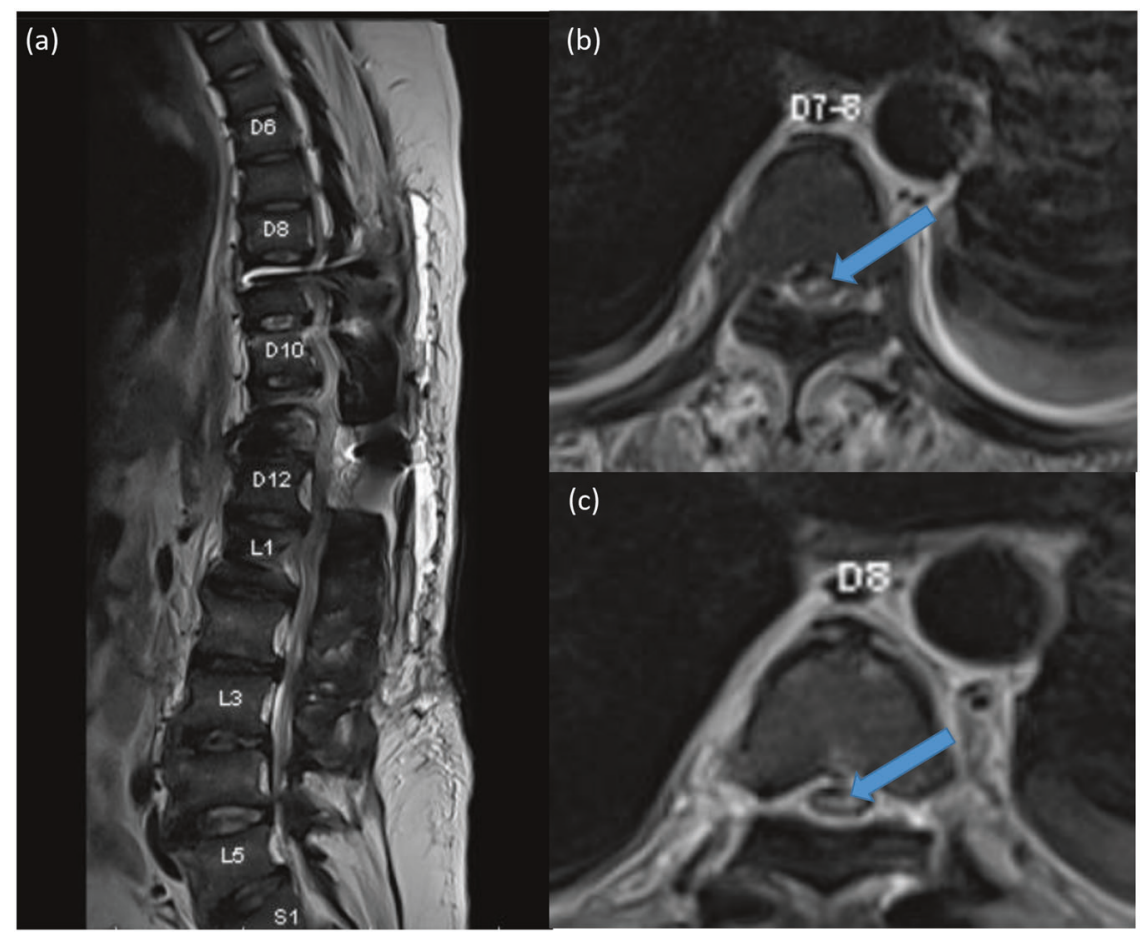

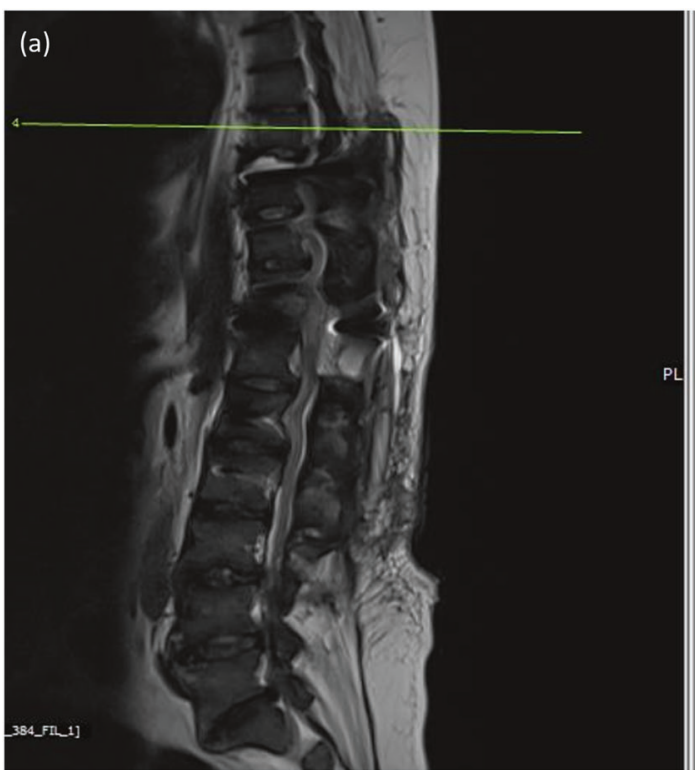

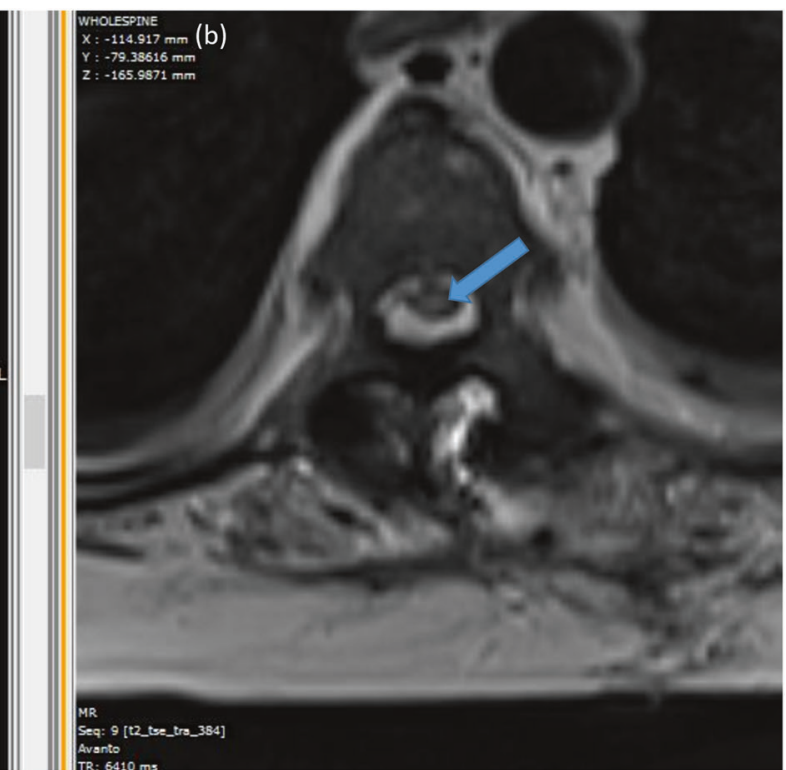

Fig. 5 Sagittal (a) and axial (b) T2 images of MRI of the patient's thoracolumbar spine taken 3 months after surgery showing the persistence of edema (marked by blue arrow) till T7 level

informed consent, the patient was taken up for surgery under neuromonitoring. The patient could not lie down straight on the operation table and hence awake fibreoptic intubation was done, anesthesia was induced and positioning was done under neuromonitoring. Posterior instrumented fusion from T9 to L2 vertebrae as well as laminectomy and decompression T11-T12 was performed. There was an incidental durotomy, which was covered with fat graft and fibrin sealant. There was no loss in the motor evoked potentials intraoperatively.

There was no deterioration of neurological status on immediate postoperative assessment. Postoperative antibiotics and analgesics were given according to the institute's protocol. Rehabilitation was initiated. On the first postoperative day, there was a significant reduction in back pain but she still complained of severe intercostal pain 
around $\mathrm{T} 10$ to $\mathrm{T} 12$ region predominantly on the left side which temporarily responded to intercostal blocks, TENS (transcutaneous electrical nerve stimulation) and Pregabalin. The intercostal blocks had to be repeated several times postoperatively. She had hyperesthesia in both lower limbs since day two for which the dose of Pregabalin was increased gradually. Postoperative MRI revealed an adequately decompressed cord (Fig. 3a, b). Radiographs and CT scan demonstrated a stable fixation (Fig. 3c, d). The screws were well positioned.

She had one spike of fever (101 degrees Fahrenheit) on day five of surgery for which no infective cause could be identified. The fever subsided with supportive treatment and antipyretic medication. At seven days post surgery, she started developing weakness and loss of sensations in both the lower limbs. Repeat MRI revealed hyper-intense signal changes extending five levels above the index level (up to $\mathrm{T} 7$ vertebra) but no static compression or instability at the index level (Fig. 4). She was provisionally diagnosed as a case of Subacute Ascending Myelopathy, a type of noncompressive myelopathy. The prognosis was discussed with the patient and attendants in detail. She was put on a high dose of intravenous methylprednisolone $(1000 \mathrm{mg}$ ) for 3 days followed by a course of oral steroids (tapering dose from 16 to $4 \mathrm{mg}$ ). There was a temporary halt in the progression of weakness. CSF tap suggested no signs of infection, virological and serological workup was negative, and MRI of the brain was normal. The wound was dry. There were no other signs of infection. Her total and differential white blood cell counts were normal and Creactive protein was normal. The weakness gradually progressed from ninth postoperative day resulting in complete paraplegia (neurological level T8) with completely absent perianal sensation and voluntary anal contraction by fourteenth postoperative day (Fig. 1d). MRI was repeated at 6 weeks and 3 months post surgery, which suggested persistent edema till T7 vertebral level (Fig. 5). She underwent a comprehensive rehabilitation program and was independent in the activities of daily living at the time of discharge on the 90th postoperative day. There was no further recovery in her neurological status even after two years of follow up.

\section{Discussion}

SPAM has been described in the literature as an unusual cause of delayed neurological deterioration after traumatic spinal cord injury in cases where the deterioration could not be explained by mechanical instability, syrinx formation or therapeutic mis-intervention. It was originally described by Frankel in 1969 [3]. Very few such cases have been reported in the literature suggesting that it is a rare entity and the condition is poorly understood [4-9]. This entity has been described only in post-traumatic cases.

Common reported features of SPAM include neurological deterioration after few days to weeks of surgical spinal stabilization, an often preceding episode of pyrexia and an area of hyperintense signal extending at least four levels above the initial injury level on T2 MRI imaging [10]. Proposed hypotheses include impairment of spinal venous drainage, arterial thrombosis, inflammatory processes or secondary injury processes. Inadvertent cord handling rarely causes extension of edema more than two levels above the injured segment and should be evident in the immediate postoperative period [3]. After an exhaustive literature search, we could not identify any case with such findings reported after atraumatic thoracic myelopathy.

The case described here had clinical and radiological features closely resembling SPAM. The fact that there was no significant trauma and no extension of edema from the index level even 6 weeks after onset of symptoms suggests that secondary injury processes are unlikely to be the cause for the condition. The gradual onset of symptoms, no sparing of the posterior column and no evidence of abnormal vascular markings on early and late MRI suggest that arterial cause is less likely [7].

Venous congestion may involve the central gray matter more than the peripheral white matter, an axial MRI finding seen in our case. However, the MRI images did not demonstrate venous stasis or engorgement of surface venous structures, findings that are frequently seen in this condition when caused by a dural arteriovenous malformation [7].

The inflammatory pathology of the disco-vertebral Anderson-like lesion, radiological findings of enthesitis, the temporal progression of myelopathy and mild pyrexia suggest that inflammatory pathology may be a likely contributing factor for ascending myelopathy [7].

Cordectomy has been described as an effective treatment by Meagher et al. in his case [4]. Laminectomy and pial incision as a treatment option has been proposed by Okada [9]. Various other treatments proposed are anticoagulation, steroids, and Mannitol. None of the treatment options proposed have been established as a standard treatment till date and the prognosis of the condition remains poor.

This case report provides us with an insight into a potential cause akin to SPAM, which needs to be considered in a patient with progressive neurological deterioration after surgical decompression and stabilization for myelopathy.

To the authors' knowledge, this is the first case report of such a condition after surgical decompression and stabilization of thoracic myelopathy.

In conclusion, ascending myelopathy is a potential cause of delayed deterioration in neurological status after surgical 
intervention. An MRI finding of cord edema extending more than four levels above the involved segments is a characteristic finding of the condition. Ascending myelopathy may lead to complete paraplegia or even tetraplegia. The precise cause of the condition remains unknown and prognosis remains poor.

Acknowledgements The authors would like to thank the concerned patient for allowing the details to be shared.

\section{Compliance with ethical standards}

Conflict of interest The authors declare that they have no conflict of interest.

Publisher's note: Springer Nature remains neutral with regard to jurisdictional claims in published maps and institutional affiliations.

\section{References}

1. Tsuzuki N, Hirabayashi S, Abe R, Saiki K. Staged spinal cord decompression through posterior approach for thoracic myelopathy caused by ossification of posterior longitudinal ligament. Spine. 2001;26:1623-30.
2. Wang H, Ma L, Xue R, Yang D, Wang T, Wang Y. The incidence and risk factors of postoperative neurological deterioration after posterior decompression with or without instrumented fusion for thoracic myelopathy. Medicine. 2016;95:e5519, https://doi.org/ 10.1097/MD.000000000000551.

3. Frankel HL. Ascending cord lesion in the early stages following spinal injury. Paraplegia. 1969;7:111-8.

4. Meagher TM, Belci M. Resolution of SPAM following cordectomy: implications for understanding pathophysiology. Spinal Cord. 2012;50:638-40.

5. Schmidt BJ. Subacute delayed ascending myelopathy after low spine injury: case report and evidence of a vascular mechanism. Spinal Cord. 2006;44:322-5.

6. Al-Ghatany M, Al-Shraim M, Levi ADO, Midha R. Pathological features including apoptosis in subacute posttraumatic ascending myelopathy. J Neurosurg Spine. 2005;2:619-23.

7. Belanger E, Picard C, Lacerte D, Lavallee P, Levi ADO. Subacute posttraumatic ascending myelopathy after spinal cord injury. $\mathrm{J}$ Neurosurg Spine. 2000;93:294-9.

8. Aito S, El Masry WS, Gerner HJ, Lorenzo ND, Pellicanò G, D'Andrea M, et al. Ascending myelopathy in the early stage of spinal cord injury. Spinal Cord. 1999;37:617-23.

9. Okada S. Sequential changes of ascending myelopathy after spinal cord injury on magnetic resonance imaging: a case report of neurologic deterioration from paraplegia to tetraplegia. Spine J. 2014;14e9-e14.

10. Yablon IG. Acute ascending myelopathy of the spine. Spine. 1989;14:11084-9. 